\title{
Simulated rat intestinal fluid improves oral exposure prediction for poorly soluble compounds over a wide dose range
}

\author{
Jörg Berghausen ${ }^{1, *}$, Frank H. Seiler ${ }^{2}$, Nathalie Gobeau ${ }^{1,3}$ and Bernard Faller ${ }^{1}$ \\ ${ }^{1}$ Novartis Institutes for Biomedical Research, Novartis Campus, CH-4002 Basel \\ ${ }^{2}$ Chemical and Pharmaceutical Profiling, Novartis Pharma AG, Novartis Campus, CH-4002 Basel \\ ${ }^{3}$ Current address: Medicines for Malaria Venture, 20, Route de Pré-Bois, 1215 Geneva 15
}

*Corresponding Author: E-mail: joerg.berghausen@novartis.com; Tel.: +41 616962335

Received: November 18, 2015; Revised: March 12, 2016; Published: March 31, 2016

\begin{abstract}
Solubility can be the absorption limiting factor for drug candidates and is therefore a very important input parameter for oral exposure prediction of compounds with limited solubility. Biorelevant media of the fasted and fed state have been published for humans, as well as for dogs in the fasted state. In a drug discovery environment, rodents are the most common animal model to assess the oral exposure of drug candidates. In this study a rat simulated intestinal fluid (rSIF) is proposed as a more physiologically relevant media to describe drug solubility in rats. Equilibrium solubility in this medium was tested as input parameter for physiologically-based pharmacokinetics (PBPK) simulations of oral pharmacokinetics in the rat. Simulations were compared to those obtained using other solubility values as input parameters, like buffer at $p H$ 6.8, human simulated intestinal fluid and a comprehensive dissolution assay based on rSIF. Our study on nine different compounds demonstrates that the incorporation of rSIF equilibrium solubility values into PBPK models of oral drug exposure can significantly improve the reliability of simulations in rats for doses up to $300 \mathrm{mg} / \mathrm{kg}$ compared to other media. The comprehensive dissolution assay may help to improve further simulation outcome, but the greater experimental effort as compared to equilibrium solubility may limit its use in a drug discovery environment. Overall, PBPK simulations based on solubility in the proposed rSIF medium can improve prioritizing compounds in drug discovery as well as planning dose escalation studies, e.g. during toxicological investigations.
\end{abstract}

\section{Keywords}

Computational ADME; preclinical pharmacokinetics; PBPK; modelling; solubility

\section{Introduction}

The use of physiologically-based pharmacokinetic (PBPK) modeling in drug development has matured and aims at prediction of plasma-concentration time profiles based on both in silico and in vitro parameters. Regardless of the advancement of the modeling software itself, the success of PBPK approaches to support decision making will strongly depend on the use of appropriate input parameters. This is particularly true in drug discovery, when the robustness of input parameters is naturally lower compared to data generated in later stage development.

A recent overview on the use of PBPK models for oral dosage forms is given by Kostewicz et al. [1]. Many reports about its use and years of application at various stages of drug development have generated 
two main strategies for implementation of PBPK in early drug discovery.

On the one hand simulation approaches can be compared to observed data as a strategy to build hypothesis regarding differences and sensitivity of input parameters. To prove or disprove such a hypothesis, results may be used to initiate further studies in order to identify the underlying reason for discrepancies between simulated and observed results. Another strategy is to use PBPK approaches to perform pharmacokinetic prediction across species, contributing to increased confidence in the design of preclinical toxicology studies and ultimately reliable prediction of human pharmacokinetics.

In order for PBPK approaches to have impact in a drug discovery setting we need to be confident of performance reliability. The quality of PBPK simulations during this early phase is often compromised by the lack of validated input data. There is greater confidence with human PBPK prediction based on the fact that at this stage of drug development, a broad set of robust input data is available. In addition, many aspects of human physiology have been studied in much greater detail compared to preclinical species. Although rodents, especially rats, are still the most relevant screening model in drug discovery, many parameters relevant for drug ADME properties are not known in detail or show a high variability. Even within one defined rat strain, a broad range of values is typically reported in the literature $[2,3]$.

It is clear that solubility can play a major role in the drug absorption process, particularly when dealing with poorly soluble compounds. As the majority of compounds in current research pipelines of pharmaceutical industry are classified into the BCS class II category [4], i.e. drug absorption is limited by solubility, it is very important to assess the development risk of such drug candidates at a very early stage of the process when choices among different chemical structures are still possible.

In order to provide PBPK simulation software with better solubility input parameters, the use of solubility values in human FaSSIF (fasted-state simulated intestinal fluid) has been proposed before. Many publications refer to human PK prediction [5], but FaSSIF has been proposed as input parameters for rats, too [6]. As rats are lacking a gall bladder, their GI tract is exposed to a constant bile flow at concentrations higher than in any other typical preclinical species. Reported bile salt concentrations in rats are in the range of 12-51 mM [7-9].

Human bio-relevant fluids are undoubtedly relevant dissolution media in order to assess the performance of drug candidates in a clinical research and development environment. However, the decision to move a potential drug candidate forward is taken during the pre-clinical phase. Up to the point of compound selection for clinical development, these compounds have only been tested in typical preclinical species, like rats, dogs, monkeys or minipigs. The role of solubility in the gastrointestinal tract of a particular species becomes evident, as soon as a compound of low solubility has shown reasonable PK parameters in rodents and is selected for testing of PK properties in a non-rodent species. Potentially, solubility may be one of the main factors that limit human bioavailability prediction based on animal studies [10], in case solubility in human GI fluids is significantly lower than in Gl fluids of preclinical species.

Solubilization of compounds in the Gl fluids is mainly driven by bile salt and phospholipid contents. To what extent differences in fluid composition influence solubility across species has been demonstrated in a comparison of solubility of drug molecules in human and dog Gl fluids [11]. For this reason, the impact of solubility on PBPK simulation results has been widely studied, especially the use of high-throughput vs. thermodynamic equilibrium solubility data. Gao et al. [6] have described a way to generate solubility input parameters for PBPK simulations of rats by applying a full $\mathrm{Gl}$ dissolution profile that takes into consideration the constant dilution by fluid secretion along the Gl transit, as well as formulation options 
that could lead to differences in supersaturation and re-precipitation behavior. However, their model is based on human FaSSIF to mimic the dilution in the rat GI fluid. Due to the sensitivity of solubility to bile salts, which is expected for many compounds in drug discovery, solubility tested in the proposed simulated rat fluid should improve the prediction power of PBPK simulations when rodents are the species of interest.

In this publication, we describe a way to generate a simulated rat intestinal fluid (rSIF) and study its impact on PBPK simulation for rats. The results are compared to simulations which use other solubility input values, like solubility in buffer $\mathrm{pH} 6.8$, FaSSIF and the dissolution curve generated by an in vitro GI dissolution assay [6]. In addition, bile salt dependent solubility estimated based on log $P$ is included in this comparison [12].

\section{Materials and Methods}

\section{Physico-chemical measurements}

Passive permeability of the compounds was investigated using the PAMPA assay [13]. In the current work, the log PAMPA value has been used, which represents the highest value of effective permeability from three measured values at $\mathrm{pH} 4, \mathrm{pH} 6.8$ and $\mathrm{pH} 8$.

Equilibrium solubility was determined using the shake-flask method using about 1.5 mg of compound. The mixture was shaken for 20 to 24 hours and the solid phase was separated by centrifugation for 15 min at 2000g. Concentration of the compound in the supernatant was determined by HPLC-UV (Agilent 1200) based on an external calibration curve.

The partition coefficient log $P$ was determined for ionizable compounds by potentiometric titration (Sirius T3, Sirius Instruments Ltd.). Non-ionizable compounds were analyzed by reverse-phase HPLC.

Determination of the acid dissociation constant ( $\mathrm{k} K \mathrm{a}$ ) was done by potentiometric titration (Sirius T3, Sirius Instruments Ltd.).

\section{Preparation of test media}

Buffer solution from Merck KGaA; pH 6.8; $0.1 \mathrm{M}$ di-sodium hydrogen phosphate / potassium hydrogen phosphate was used.

Preparation of human FaSSIF-V2 was done based on the suggestion by Jantratid et al. [14]. To obtain one liter of clear FaSSIF-V2, sodium taurocholate and sodium chloride were first dissolved in $400 \mathrm{ml}$ of purified water, followed by the addition of $1 \mathrm{ml}$ of $1 \mathrm{M} \mathrm{HCl}$. After stirring for $30 \mathrm{~min}$, lecithin was added and the mixture was sonicated until complete dissolution and stirred for another two hours. Then, maleic acid and $500 \mathrm{ml}$ of water were added. After overnight stirring, the $\mathrm{pH}$ was adjusted to 6.5 by addition of $1 \mathrm{M}$ $\mathrm{NaOH}$ and the total volume was adjusted to $1 \mathrm{~L}$.

\section{Simulated rat intestinal fluid}

A rat simulating intestinal fluid (rSIF) was developed as surrogate to mimic intestinal conditions in vivo with regard to bile salt and phospholipid concentrations, surface tension, $\mathrm{pH}$, buffer capacity and osmolarity [15]. This was done to supplement previously published human [14] and canine [11] media. Due to unavailability of sufficient ex vivo samples, published information was used to define the target properties of rSIF. Table 1 compares the main properties of rSIF with human FaSSIF-V2. 
Table 1. Main properties of rat simulated intestinal fluid (rSIF) in comparison to human FaSSIF-V2

\begin{tabular}{|c|c|c|}
\hline & rSIF & FaSSIF-V2 \\
\hline $\mathrm{pH}$ & 6.0 & 6.5 \\
\hline Buffer capacity $[\mathrm{mmol} / \mathrm{I} / \mathrm{pH}]$ & 15.2 & 10 \\
\hline Surface Tension $[\mathrm{mN} / \mathrm{m}]$ & 42 & 54.3 \\
\hline Osmolality $[\mathrm{mOsmol} / \mathrm{kg}]$ & 480 & 180 \\
\hline Bile Salt Concentration $[\mathrm{mM}]$ & 25 & 3 \\
\hline Phospholipid Concentration $[\mathrm{mM}]$ & 5.16 & 0.2 \\
\hline
\end{tabular}

Due to the constant bile flow in rats due to a lack of a gall bladder and most typical housing conditions with constant access to food, a homostatic condition was assumed for rats. In consequence, fasted and fed states were not distinguished for the rat medium.

Rat intestinal pH was averaged to 6.0 based on values reported elsewhere [2,16,17]. An average was selected to reflect the main areas of absorption within the proximal intestine with reported $\mathrm{pH}$ values between 5.0 and 7.1. To allow higher throughput, use of multiple pH media was neglected.

Adjustment of surface tension had to be balanced with bile salt and phospholipid concentrations. Addition of cholic acid and chenodeoxycholic acid sodium was required to achieve targeted surface tension values. It was found that combination of bile salts provided a synergistic effect and provided best reduction of surface tension. Both substances are reported to be present in rat bile fluids and thus have physiological relevance [18]. Surface tension values obtained for $\mathrm{rSIF}$ are in line with recent data [19]. Bile salt and phospholipid concentrations are slightly above values referenced and used for example in GastroPlus models and other references [17]. The values for buffer capacity were estimated taking into account human FaSSIF-V2 and the constant bile flow, as there was no reference available at the time of rSIF development. Recent data from Merchant [19] indicate that this value appears to be too low.

Depending on new insights rSIF may be further refined with regard to bile salt concentration, buffer capacity and osmolality. Further refinement of media could also reflect more section specific $\mathrm{pH}$ and bile concentrations in analogy to human media published [14].

Table 2. Composition of rSIF; $100 \mathrm{ml}$ target volume

\begin{tabular}{|c|c|c|c|}
\hline & Conc. $(\mathrm{mM})$ & Molecular weight $(\mathrm{g} / \mathrm{mol})$ & Weighted sample $(\mathrm{mg})$ \\
\hline Sodium taurocholate & 5.00 & 537.7 & 269 \\
\hline $\mathrm{NaCl}$ & 18.70 & 58.0 & 108 \\
\hline Lecithin & 5.16 & 775.0 & 400 \\
\hline Maleic acid & 29.86 & 116.1 & 347 \\
\hline Purified water & - & - & q.s. \\
\hline $\mathrm{NaOH}$ 1 N & - & - & 8 \\
\hline Sodium oleate & 0.26 & 304.4 & 538 \\
\hline Sodium cholate hydrate & 12.50 & 430.6 & 311 \\
\hline $\begin{array}{c}\text { Sodium } \\
\text { chenodeoxycholic acid }\end{array}$ & 7.50 & 414.6 & 60 \\
\hline Glyceryl Monooleate & 1.67 & 356.5 & \\
\hline
\end{tabular}




\section{Preparation of rSIF}

In order to prepare $100 \mathrm{ml}$ of rSIF, a composition according to Table 2 was used. Sodium taurocholate, sodium cholate hydrate, sodium chenodeoxycholic acid and $\mathrm{NaCl}$ were dissolved in $50 \mathrm{ml}$ of purified water. After stirring for $30 \mathrm{~min}$, lecithin, sodium oleate and glyceryl monooleate were added. Subsequently, the mixture was sonicated for about 5-10 min and stirred overnight. A clear solution was obtained. Maleic acid and $40 \mathrm{ml}$ of purified water were added, resulting in a suspension of $\mathrm{pH}$ around 2.2. The $\mathrm{pH}$ was adjusted to 6.0 by $1 \mathrm{M} \mathrm{NaOH}$. The mixture was stirred until a clear solution was obtained. If needed, the $\mathrm{pH}$ was adjusted, again. Finally, the volume was filled up to $100 \mathrm{ml}$ by addition of purified water.

The surface tension was determined in triplicates using a Kibron delta-8 tensiometer (Kibron Inc., Helsinki). An average value of $42.8 \mathrm{mN} / \mathrm{m}$ was obtained.

\section{PBPK simulations}

PBPK simulations were performed using the ACAT model of the GastroPlus software (Simulations Plus Inc., Lancaster) version 8.6.

The distribution and elimination were described by a compartmental PK model. The number of compartments and parameters of the model were obtained by fitting the in vivo i.v. time-concentration profiles in the PKPlus module of Gastroplus.

The absorption was predicted by the ACAT model in Gastroplus using the "rat physiological fasted" GI tract and based on the physico-chemical and in vitro ADME properties of the compounds.

The permeability of the compound was characterized by measurement in PAMPA. First, a calibration of the PAMPA assay to the Gastroplus permeability $P_{\text {eff }}$ input parameter was carried out. It was based on the curve of PAMPA versus human fraction absorbed for 83 marketed compounds. The curve of the Gastroplus intrinsic permeability $P_{\text {eff }}$ versus human fraction absorbed was obtained by varying the $P_{\text {eff }}$ value, see Figure 1. The correlation between PAMPA and $P_{\text {eff }}$ was obtained by matching the two curves.

To be consistent with other data of internal databases an animal weight of $250 \mathrm{~g}$ was used. This is slightly below the actual weight of animals in the current study. The difference in simulation outcome has been tested for selected cases (data not shown) and is rather small. No effect on the ranking of the simulation results has been observed. The animals had free access to food, thus the feeding state was uncontrolled, neither fasted nor fed. Due to the lack of the gall bladder, the difference between fasted and fed state for rats is regarded as to be small. As tested (data not shown), the impact of physiology of the simulation model is marginal in comparison to the solubility impact. Thus, the fasted rat physiology was used.

The Absorption Scaling Factor model, designed to take into account the regional changes in permeability, was the "Opt $\log D$ Model SA/V 6.1". The scaling coefficient C4 in the colon was set to zero in order to avoid overestimation of colonic absorption as outlined in equation (1):

$$
A S F=C 3 \cdot 10^{C 4 \log D}
$$


a) PAMPA assay for 83 marketed compounds

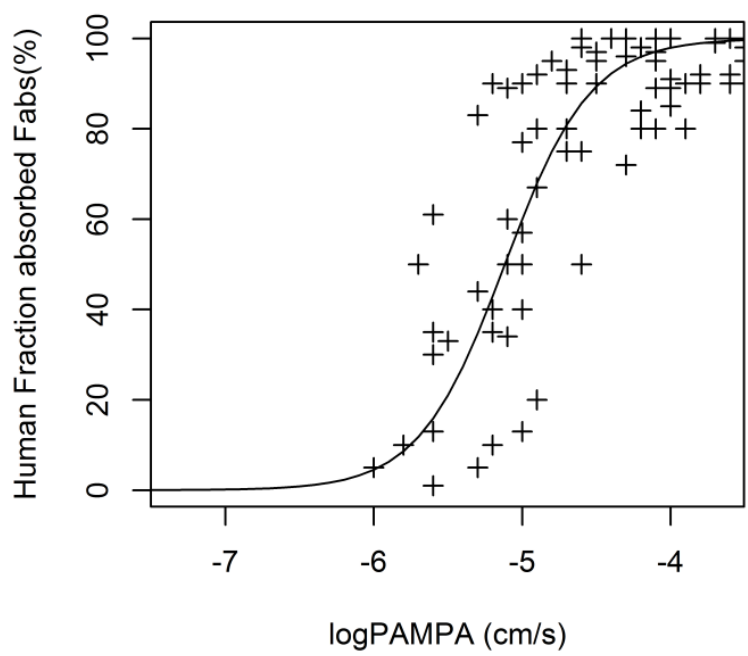

c) PAMPA vs Gastroplus

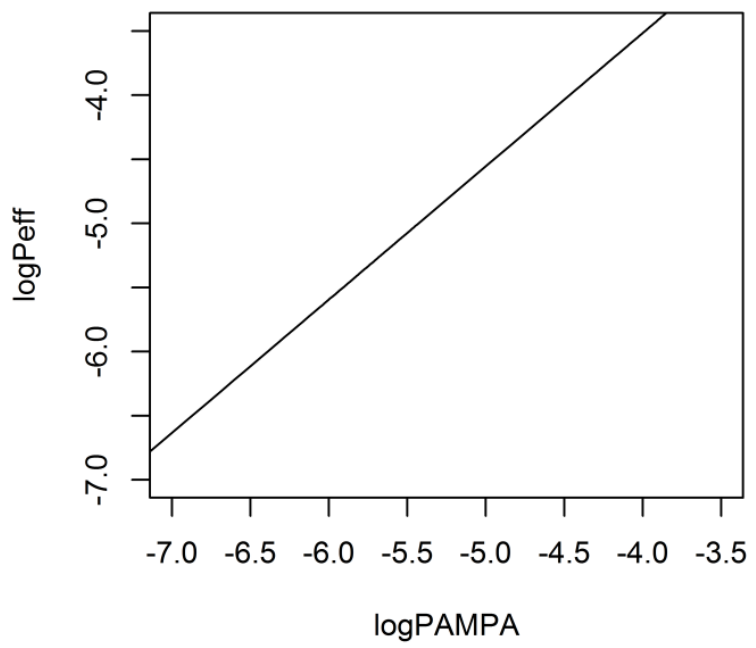

b) Gastroplus predictions

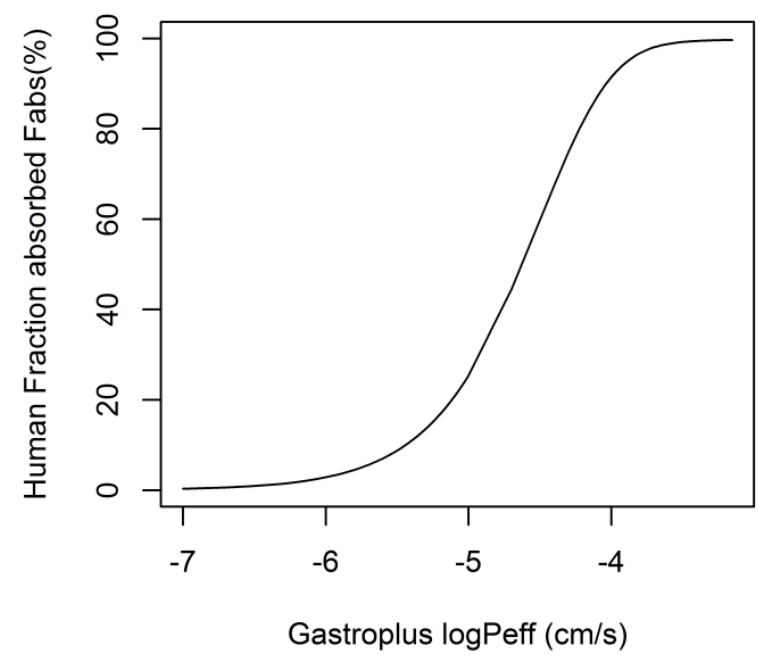

a) PAMPA: Fabs $=\frac{1}{1+\left(\frac{7.6310^{-6}}{\text { P }_{\text {PAMPA }}}\right)^{1.5}}$

b) Gastroplus: Fabs $=\frac{1}{1+\left(\frac{1.510^{-5}}{P_{\text {eff }}}\right)^{1.4}}$

c) $\log \mathrm{P}_{\text {eff }}=0.48+1.039 \times \log \mathrm{P}_{\mathrm{PAMPA}}$

Figure 1: $P_{\text {eff }}$ conversion based on log PAMPA measurements

As a consequence, the original model led to high absorption for all lipophilic drugs regardless of their PAMPA (or Caco-2) permeability, which did not match our observations. The property space of the training set used in the GastroPlus software where poorly permeable compounds are typically hydrophilic molecules does not match the property space of our discovery molecules that are characterized by a higher average molecular weight and for which poor permeability is not exclusively driven by low lipophilicity.

The liver first-pass extraction was estimated with the well-stirred model based on in vitro microsomal clearance without taking into account plasma protein binding or microsomal binding. This approach was found to be reasonable by Parrot et al [12], Germani et al [21] and Jones et al [22]. It was compared with the estimation based on the in vivo clearance and provided better predictions (see Figure 2). 


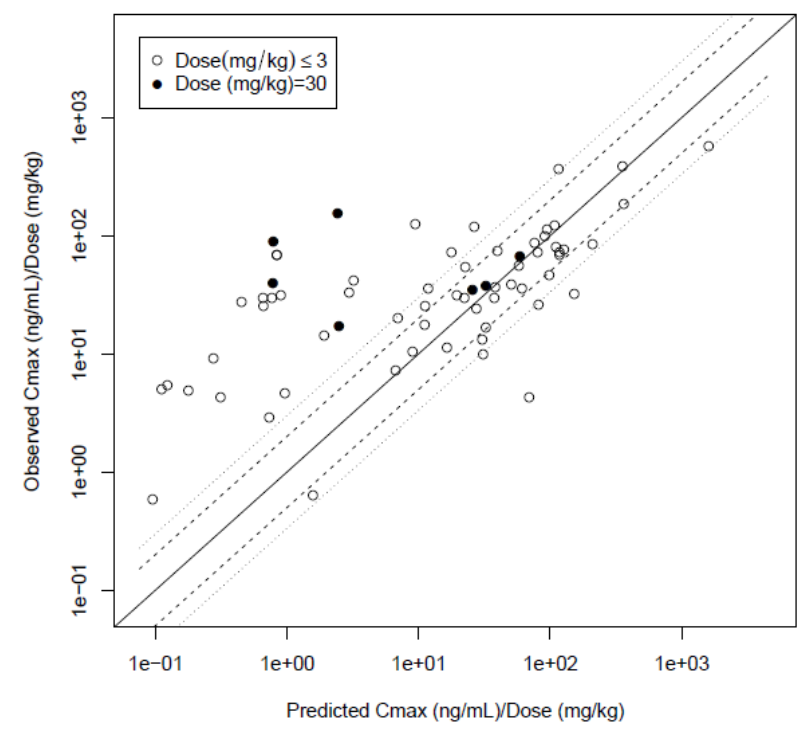

a) Liver first pass based on in vivo clearance

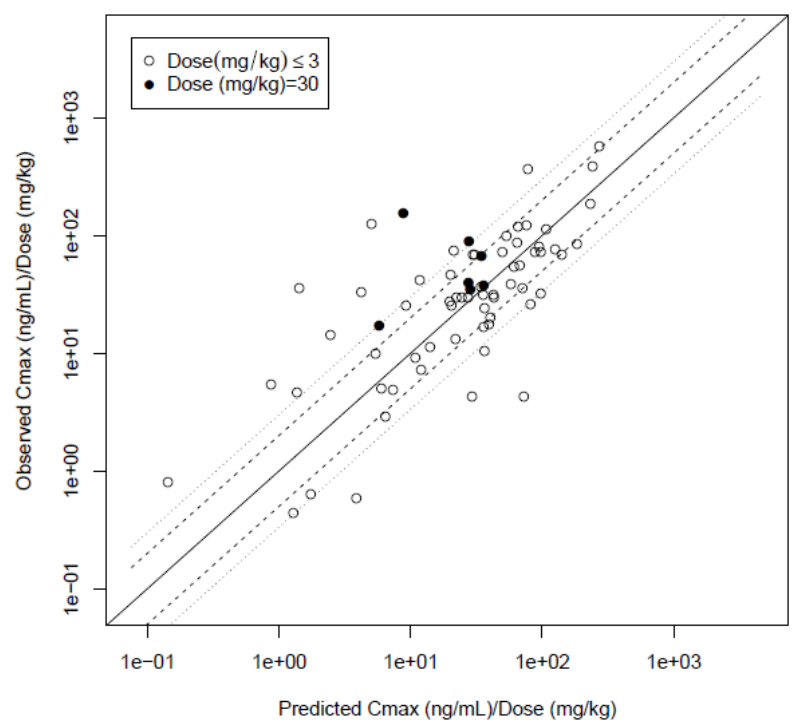

b) Liver first pass based on microsomal clearance

Figure 2: Comparison of the performance of the model between the estimation of the liver first pass with the in vivo clearance (a) and microsomal clearance (b) for 61 proprietary compounds administered to rats.

The octanol-water partition coefficient $\log P$ is based on measured values. For all suspensions, a particle radius of $7 \mu \mathrm{m}$ was assumed, which is a typical number based on our experience with compounds in the lead optimization phase. Typically, the unknown solid state and the impact of the amorphous form are supposed to play a major role for particle dissolution, which may overrule the particle size effect.

Information about dose dependence of systemic clearance is typically not available in the lead optimization stage and the assumption made in this study is that clearance does not significantly change with the dose.

\section{Pharmacokinetic studies}

Pharmacokinetic studies have been performed in male Sprague-Dawley rats, originating from Charles River Wiga (Germany). The experiment was performed according to the regulations effective in the Canton Basel-City, Switzerland.

Six to four days before first drug administration, the rats (body weight approx. 270-330 g) were anesthetized and catheters were surgically implanted into the femoral artery (for blood collection) and femoral vein (for intravenous injection). The catheters were exteriorized at the neck where they were fixed via the tether and a flexible spring to a Harvard swivel system, which allowed blood sampling and intravenous injections without disturbing the freely-moving animal. For analgesic treatment, animals received Temgesic $\AA$ (10 $\mu \mathrm{g} \mathrm{kg}^{-1}$ S.c.) before surgery and subsequently twice at appropriate times after surgery. Animals were kept individually in Macrolon cages, with free access to food and water throughout the experiment.

Dosing i.v. and p.o. was performed in two different animal groups. For per oral administration, the compounds were formulated as a suspension in methyl cellulose. For intravenous administration, the compounds were dissolved in an appropriate mixture of N-methyl pyrrolidone (NMP) and poly(ethylene glycol) 200 (PEG). For sample analysis, approximately $50 \mu$ of blood was taken. $30 \mu \mathrm{L}$ of blood was mixed with $200 \mu \mathrm{L}$ of acetonitrile and centrifuged at $4{ }^{\circ} \mathrm{C}$. Approx. $200 \mu \mathrm{L}$ of supernatant was transferred into a microtiter plate and mixed with $200 \mu \mathrm{L} 0.1 \%$ formic acid. An aliquot of each sample was injected into the 
LC-MS/MS system for analysis.

\section{Gl dissolution assay}

Full GI dissolution was performed by a modified setup based on the proposal of Gao et al. [6]. Their GI dissolution model takes into consideration the transit times in the stomach and four additional segments in the intestine. Gao et al. included the dilution that is supposed to happen during the transit through the gastrointestinal tract. In particular, dilution was based on basal acid output, the pancreatic juice secretion rate and the bile flow. Gao et al. proposed a basal acid output of $0.8657 \mathrm{ml} / 4 \mathrm{~h} / 100 \mathrm{~g}$. In order to calculate the dilution in the small intestine, a pancreatic juice secretion rate of $0.033 \mathrm{ml} / \mathrm{min} / \mathrm{kg}$ is suggested; bile flow is set to $70 \mathrm{ml} / \mathrm{day} / \mathrm{kg}$ in their setup. The corresponding transit times are $0.25 \mathrm{~h}$ in the stomach and $0.2,2.0,4.5$ and $8 \mathrm{~h}$ for duodenum, jejunum/ileum, cecum and colon, respectively. Dilution in the stomach is supposed to be caused by basal acid output only. In the small intestine, the basal acid output adds to the secretion of pancreatic juice and bile. Based on these assumptions, dilution factors of 1.5 for the stomach, 1.9 for the duodenum, 5.8 for jejunum/ileum and 2.9 for the cecum were proposed. No further dilution was considered in the colon, since most fluid had been absorbed.

For improved differentiation of weakly basic compounds in our setup, the $\mathrm{pH}$ of the stomach compartment was lowered to $\mathrm{pH} 3$. As dilution medium the rSIF as proposed in Table 2 was used instead of FaSSIF-V2.

\section{Results and Discussion}

In vitro solubility studies

In order to study the difference of equilibrium solubility in the proposed rat fluid (rSIF) in comparison to buffer solubility and human FaSSIF-V2, a set of proprietary compounds has been investigated by the shakeflask method. The compounds have been selected based on availability of in vivo data, and sufficient compound amount for running solubility and GI dissolution studies. Also, only compounds exhibiting high in vitro permeability have been chosen, thus keeping the impact of permeability on drug absorption low.

Solubility results in buffer $\mathrm{pH}$ 6.8, human FaSSIF-V2 and rSIF media for the selected compounds are summarized in Table 3.

Table 3. Solubility (g/L) of selected compounds in buffer $\mathrm{pH}$ 6.8, FaSSIF-V2 in comparison to rSIF

\begin{tabular}{|c|c|c|c|}
\hline & buffer pH 6.8 & FaSSIF-V2 & rSIF \\
\hline Compound A & 0.001 & 0.0041 & 0.105 \\
\hline Compound B & 0.004 & 0.0001 & 0.448 \\
\hline Compound C & 0.031 & 0.043 & 0.711 \\
\hline Compound D & 0.005 & 0.003 & 0.023 \\
\hline Compound E & $<0.0005$ & 0.0009 & 0.046 \\
\hline Compound F & 0.05 & 0.14 & 0.881 \\
\hline Compound G & 0.003 & 0.013 & 0.193 \\
\hline Compound H & 0.0002 & 0.0005 & 0.01 \\
\hline Compound I & 0.0025 & 0.002 & 0.015 \\
\hline
\end{tabular}

Mostly compounds exhibiting low solubility in both buffer pH 6.8 and FaSSIF-V2 have been selected. Solubility in rSIF can differ vastly from the other two media investigated, thus leading to the assumption 
that FaSSIF-V2 solubility is not able to provide a realistic input parameter for PBPK simulations in the rat. Compound $\mathrm{F}$ has been selected as an example of acceptable solubility in FaSSIF-V2, but rSIF still showing a 7-fold higher equilibrium value. This result will be used as a test case whether solubility in rSIF would lead to over-prediction in PBPK simulations.

\section{Bile salt dependent solubility}

There is little doubt that the use of thermodynamic solubility values in buffers in many cases will not be very useful to predict in vivo concentration-time profiles. With the exception of well soluble compounds with good wetting properties, buffer solubility values will most likely underestimate the in vivo solubilization of most molecules. Since the introduction of the human FaSSIF and FeSSIF media, there is an option to obtain solubility values which are more appropriate as input parameters for PBPK simulations and FaSSIF solubility maybe one of the physico-chemical parameters that is determined as one of the first after a compound is sent out for characterization.

However, to properly assess the risk for clinical development it is necessary to understand the impact of solubility on in vivo absorption during lead optimization and thus in preclinical species. Although the FaSSIF and FeSSIF media allow studying the potential solubility limitation for absorption in humans, they may not be appropriate input parameters in preclinical species. The bile salt concentration in gastrointestinal fluids in the fasted dog is known to be at the level of human FeSSIF rather than FaSSIF [11], and the situation in rats is substantially different. Due to the absence of a gall bladder and thus a constant bile flow, factors limiting absorption in a rat may be rather different as compared to the situation in humans. As bile salt concentrations in rat $\mathrm{Gl}$ fluids are reported up to a level of $51 \mathrm{mM}$ [9] the rat is likely to be a better solubilizer for lipophilic compounds which might be better absorbed than in higher species.

The GastroPlus software includes an estimation of bile salt dependent solubility based on log $P$ according to the equation of Mithani et al. [20]:

$$
S R=\frac{S C_{b s}}{S C_{a q}}
$$

where $S C_{b s}$ is defined as the solubilization capacity of bile salt (moles drug/mole bile salt) and $S C_{a q}$ is defined as the solubilization capacity of water (moles drug/mole water).

For their set of compounds, they found:

$\log S R=2.23+0.61 \log P$

This approach was evaluated based on a dataset of proprietary compounds. For these compounds, buffer solubility and the solubility in buffer containing various amount of sodium taurocholate was determined, see Table 4 . To calculate $S C_{b s}$, the molar solubility at a defined bile salt concentration was divided by the actual bile salt concentration, i.e. 10,50 or $100 \mathrm{mM}$ in our data set. $S C_{\text {aq, }}$, correspondingly, is obtained by dividing the molar solubility in buffer $\mathrm{pH} 6.8$ by $55.5 \mathrm{~mol} / \mathrm{liter}$.

Calculation of the $\log P$ dependence of the solubility ratio $(S R)$ as defined by Mithani et al. led to a linear regression similarly to the equation as proposed by Mithani, see Figure 3 . While this data principally 
supports the linear dependency of $S R$ on $\log P$, this equation is based on a fit through several orders of magnitude for both $\log P$ and $\log S R$, which will compromise the ability to predict individual solubility values with the accuracy required for PBPK modeling. In order to estimate the predictive power of the Mithani equation, the calculated values and the measured solubility at different bile salt concentrations are displayed in Figure 3. For some of the compounds of the test set (see Table 4) the variation in $S R$ can be more than one order of magnitude within the different concentration of bile salts used to determine the solubility, i.e. 10,50 or $100 \mathrm{mM}$. The maximum difference to the linear fit is found to be about $2 \log$ units of $S R$. Importantly, the differences to the linear fit do not appear to be markedly log $P$ dependent. Also, the increase in solubility with increasing bile salt concentration for many compounds is not a linear dependency, see Table 4.

Table 4. Solubility test set for taurocholate concentrations of 10,50 and $100 \mathrm{mM}$. S indicates solubility in buffer at pH 6.8. Calculated solubility ratio according to taurocholate concentrations (log SR10, $\log$ SR50, $\log$ SR100), based on the Mithani equation (1).

\begin{tabular}{|c|c|c|c|c|c|c|c|c|c|c|}
\hline & $M_{\mathrm{w}}$ & Log $P$ & $\begin{array}{c}\mathrm{pKa} \\
\mathrm{acid} / \mathrm{b} a s e\end{array}$ & S/mM & $10 \mathrm{mM}$ & $50 \mathrm{mM}$ & $100 \mathrm{mM}$ & $\begin{array}{c}\text { Log } \\
\text { SR10 }\end{array}$ & $\begin{array}{c}\text { Log } \\
\text { SR50 }\end{array}$ & $\begin{array}{c}\text { Log } \\
\text { SR100 }\end{array}$ \\
\hline Cpd 1 & 529.5 & 5.2 & $5.4 \mathrm{~b} ; 3.9 \mathrm{~b}$ & 0.0019 & 17.78 & 483.2 & 666.9 & 7.718 & 8.453 & 8.292 \\
\hline Cpd 2 & 379.4 & 3.2 & $3.4 \mathrm{~b}$ & 0.022 & 0.04 & 0.26 & 0.34 & 4.004 & 4.118 & 3.933 \\
\hline Cpd 3 & 560.5 & 4.1 & $8.2 \mathrm{~b} ; 3.7 \mathrm{~b}$ & 0.0054 & 0.42 & 3.95 & 5.49 & 5.639 & 5.913 & 5.755 \\
\hline Cpd 4 & 558.2 & 4.6 & $9.1 \mathrm{~b} ; 4.1 \mathrm{~b}$ & 0.001 & 0.7 & 4.12 & 4.07 & 6.589 & 6.660 & 6.354 \\
\hline Cpd 5 & 577.8 & 4.9 & $9.1 \mathrm{~b} ; 3.9 \mathrm{~b}$ & 0.3202 & 10.8 & 11.02 & 11.23 & 5.272 & 4.582 & 4.289 \\
\hline Cpd 6 & 410.4 & 2.6 & $5.1 \mathrm{~b}$ & 0.0975 & 2.27 & 4.35 & 9.86 & 5.111 & 4.695 & 4.749 \\
\hline Cpd 7 & 485.5 & 4.3 & $4.2 \mathrm{~b}$ & 0.001 & $<0.001$ & 0.15 & 0.34 & - & 4.907 & 4.962 \\
\hline Cpd 8 & 453.6 & 3.1 & $3.7 \mathrm{~b}$ & 0.0066 & 0.01 & 0.01 & 0.01 & 3.924 & 3.225 & 2.924 \\
\hline Cpd 9 & 710.7 & 7.1 & $5.11 \mathrm{a} ; 3.8 \mathrm{~b}$ & 0.0014 & 0.03 & 1.27 & 1.9 & 5.073 & 6.001 & 5.875 \\
\hline Cpd 10 & 313.4 & 3.7 & n.d. & 0.16 & 0.14 & 1.6 & - & 3.686 & 4.045 & - \\
\hline Cpd 11 & 361.3 & 3.9 & $11.7 \mathrm{a}$ & 0.14 & 0.46 & 0.51 & 0.63 & 4.261 & 3.607 & 3.398 \\
\hline
\end{tabular}

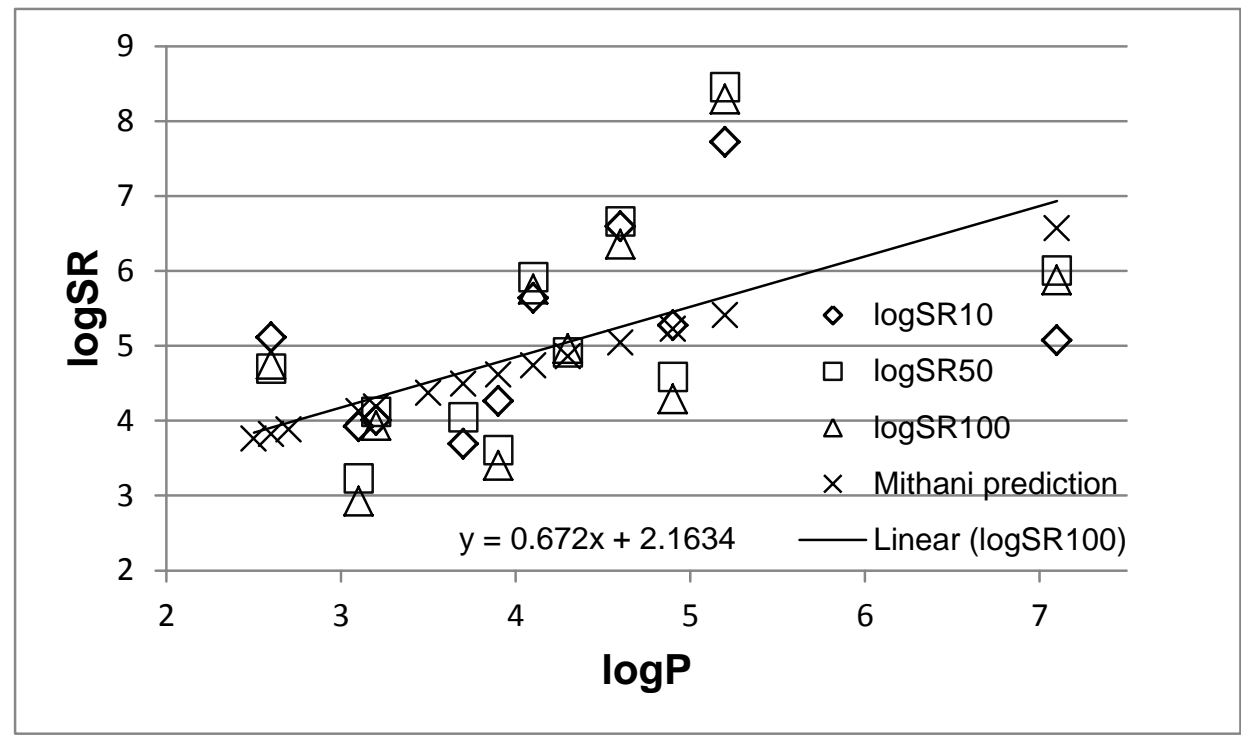

Figure 3. $\log P$ dependence of solubility ratio at $10 \mathrm{mM}(\log S R 10), 50 \mathrm{mM}(\log S R 50)$ and $100 \mathrm{mM}(\operatorname{logSR} 100)$ taurocholate concentration. The linear fit is based on log SR100, the crosses represent the prediction according to equation (2).

As a consequence, due to the significant deviations from the linear fit for many compounds, the estimation of bile salt solubility for individual compounds is regarded as not sufficient to support studying 
the impact of solubility in PBPK simulations. The estimation of $\log P$ dependent solubilization ratio might be justified in cases where no bile salt dependent solubility can be determined, however, there remains a risk of being misled. This is especially true for low soluble compounds, as changes of solubility by only a factor 2 to 4 might strongly impact the absorption profile of a compound. Thus, FaSSIF solubility will not provide accurate enough information to further extrapolate the contribution of bile salt solubilization at concentrations that are assumed to mimic the in vivo situation in rats.

\section{PBPK Simulations}

The importance of solubility input parameters for PBPK simulations of low soluble compounds is indisputable. At low dose, a significant portion of a compound administered might be dissolved in the stomach and subsequently be absorbed. However, at a higher dose, there is a larger portion remaining undissolved. Thus, the solubilization power of the intestinal fluid is more relevant for the absorption of higher doses. As a consequence, GI dissolution profiles adapted to the relevant species should positively impact the prediction results, especially for Cmax. To test this hypothesis, GastroPlus simulations have been performed at different dose levels and simulation results are compared to in vivo results.

Compounds with no expected permeability limitation according to in vitro data (PAMPA) and low to moderate total blood clearance have been selected to investigate the impact of solubility parameters on PBPK simulations, see Table 5. A systematic approach was chosen to compare the impact of solubility on the simulation results for the selected compounds. Five different solubility input parameters have been chosen. First, the thermodynamic equilibrium solubility in buffer was used, without correction for bile salt solubilization. In a next step, the FaSSIF solubilty value was used to calculate the solubilization ratio (SR). Based on the data in Table 4, the theoretical $S R$ calculation as provided by the GastroPlus software was tested in another approach. The rat SIF fluid solubility was used without any correction for bile salt content, similar to the thermodynamic solubility value. According to the authors' experience, correction of bile salt dependent solubility using the built-in in vitro $S R$ calculation compromises the value of the solubility in the proposed rat SIF medium. Finally, if not limited by compound availability, the full dissolution curve was recorded to mimic the dissolution properties of the compound during its transit through the rat gastrointestinal tract, based on the modified procedure of Gao et al.[6].

Table 5. Summary of input parameters for PBPK simulations. First pass extraction ratio (ER\%) based on rat liver microsomes. Clearance as a result of fit to in vivo data by the PKPlus module. The volume of distribution into the respective distribution model is given by $V_{c}, V_{2}$ and $V_{3}$. In case only two numbers are given, they refer to $V_{c}$ and $V_{2}$, respectively.

\begin{tabular}{|l|c|c|c|c|c|c|c|}
\hline & $\begin{array}{c}\text { First pass } \\
\text { ER\% }\end{array}$ & $\mathrm{pKa}$ & & $\begin{array}{c}\text { Log } \\
\text { PAMPA } / \\
\mathrm{cm} / \mathrm{s}\end{array}$ & Log $P$ & $\begin{array}{c}\text { Clearance, } \\
\text { L/h/kg }\end{array}$ & $V_{\mathrm{c}}, V_{2}, V_{3}$ \\
\hline Compound A & 77 & 3.8 & Base & -3.9 & 1.7 & 0.42 & $0.38,0.15,1.67$ \\
\hline Compound B & 28 & 5.4 & Acid & -4.0 & 3.7 & 1.10 & $3.28,4.58$ \\
\hline Compound C & 92 & $<3$ & base & -3.6 & 3.6 & 3.48 & $2.77,0.95,1.74$ \\
\hline Compound D & 25 & 4.7 & Base & -3.6 & 3.5 & 0.98 & $4.32,3.50,17.62$ \\
\hline Compound E & 56 & 2.7 & Base & -3.5 & 4.2 & 0.42 & $0.88,2.19$ \\
\hline Compound F & 67 & 3.2 & Base & -3.4 & 3.1 & 0.42 & $0.46,0.47$ \\
\hline Compound G & 55 & 3.4 & Base & -4.6 & 3.9 & 3.36 & $4.50,0.80$ \\
\hline Compound H & 38 & 3.5 & Base & -4.5 & 5.3 & 1.34 & $3.74,7.19$ \\
\hline Compound I & 37 & 1.7 & Base & -4.2 & 2.8 & 5.19 & $2.57,0.55$ \\
\hline
\end{tabular}


The PBPK simulation results based on the ACAT model have been split into lower and higher dose studies and are summarized in Tables 6 to 9. Simulation results are compared to in vivo data from compounds dosed orally as suspension.

\section{PBPK results}

Lower range of p.o. doses

The impact of various solubility input parameters on PBPK simulation results has been tested. As evaluation criteria for the success of PBPK simulations versus the in vivo PK results, $C_{\max }$ and AUC were chosen, see Table 6 and Table 7. Different solubility input parameters have been tested, as described above (see Table 3). In addition, the full Gl dissolution profile at the respective dose has been measured and the solubility values for each compartment have been entered according to the method section.

Table 6. PBPK results of $C_{\max }$ in $\mu \mathrm{g} / \mathrm{mL}$ for lower dose range based on various solubility input. $C_{\max }$ buffer, FaSSIF, FaSSIF with theoretical bile salt correction ( $C_{\max }$ FaSSIF bile) and rSIF refer to equilibrium values, whereas $\mathrm{GI}$ disso comprises the input of the full GI dissolution profile. Pred. vs. obs. denotes the ratio of the predicted values vs. the observed $C_{\max }$.

\begin{tabular}{|c|c|c|c|c|c|c|c|}
\hline & $\begin{array}{l}\text { Dose } \\
\mathrm{mg} / \mathrm{kg}\end{array}$ & $C_{\max }$ obs & $\begin{array}{c}C_{\max } \\
\text { buffer }\end{array}$ & $\begin{array}{c}C_{\max } \\
\text { FaSSIF }\end{array}$ & $\begin{array}{c}C_{\max } \\
\text { FaSSIF } \\
\text { bile }\end{array}$ & $C_{\max } \mathrm{rSIF}$ & $\begin{array}{c}C_{\max } \mathrm{Gl} \\
\text { disso }\end{array}$ \\
\hline Compound A & 10 & 5.041 & 0.035 & 0.273 & 0.089 & 2.185 & \\
\hline Pred. vs. obs. & & & 0.0069 & 0.0542 & 0.0175 & 0.4333 & \\
\hline Compound B & 3 & 0.138 & 0.012 & 0.012 & 0.040 & 0.238 & \\
\hline Pred. vs. obs. & & & 0.0869 & 0.0869 & 0.2896 & 1.7246 & \\
\hline Compound C & 3 & 0.134 & 0.028 & 0.043 & 0.046 & 0.045 & \\
\hline Pred. vs. obs. & & & 0.2089 & 0.3208 & 0.3432 & 0.3358 & \\
\hline Compound D & 3 & 0.213 & 0.053 & 0.057 & 0.124 & 0.167 & 0.204 \\
\hline Pred. vs. obs & & & 0.2488 & 0.2676 & 0.5822 & 0.7840 & 0.9577 \\
\hline Compound E & 10 & 2.211 & 0.069 & 0.071 & 0.609 & 1.502 & 1.517 \\
\hline Pred. vs. obs & & & 0.0312 & 0.0321 & 0.2754 & 0.6793 & 0.6861 \\
\hline Compound F & 10 & 3.753 & 2.743 & 2.743 & 2.742 & 2.743 & 2.308 \\
\hline Pred. vs. obs & & & 0.7309 & 0.7309 & 0.7306 & 0.7309 & 0.6150 \\
\hline Compound G & 3 & 0.020 & 0.004 & 0.030 & 0.022 & 0.074 & 0.073 \\
\hline Pred. vs. obs & & & 0.2000 & 1.500 & 1.100 & 3.700 & 3.650 \\
\hline Compound $\mathrm{H}$ & 3 & 0.082 & 0.0007 & 0.0007 & 0.031 & 0.037 & 0.071 \\
\hline Pred. vs. obs & & & 0.0085 & 0.0085 & 0.3780 & 0.4512 & 0.8659 \\
\hline Compound I & 3 & 0.056 & 0.011 & 0.008 & 0.020 & 0.062 & \\
\hline Pred. vs. obs & & & 0.1964 & 0.1429 & 0.3571 & 1.107 & \\
\hline
\end{tabular}

Results for the lower dose range (see Table 6, Table 7 and Figure 4) support the assumption that the buffer solubility typically leads to an underprediction of both $C_{\max }$ and AUC. The only exception is compound $\mathrm{F}$, despite the fact that its solubility in rSIF is much higher than in buffer and FaSSIF-V2 media. Apparently, its rather high permeability and sufficient solubility lead to nearly complete absorption, yet if the lowest measured solubility value is used. Even a ten times higher solubility value, according to rSIF in vitro results, does not change the predicted concentration-time profile for this compound for the $10 \mathrm{mg} / \mathrm{kg}$ dose. 
Table 7. PBPK results of AUC (0-t) in $\mu \mathrm{g} \mathrm{h} / \mathrm{mL}$ for lower dose range based on various solubility input. AUC buffer, FaSSIF, FaSSIF with theoretical bile salt correction (AUC FaSSIF bile) and rSIF refer to equilibrium values, whereas GI disso comprises the input of the full GI dissolution profile. Pred. vs. obs. denotes the ratio of the predicted values vs. the observed AUC.

\begin{tabular}{|l|c|c|c|c|c|c|c|}
\hline & $\begin{array}{c}\text { Dose, } \\
\mathrm{mg} / \mathrm{kg}\end{array}$ & AUC obs & $\begin{array}{c}\text { AUC } \\
\text { buffer }\end{array}$ & $\begin{array}{c}\text { AUC } \\
\text { FaSSIF }\end{array}$ & $\begin{array}{c}\text { AUC } \\
\text { FaSSIF } \\
\text { bile }\end{array}$ & AUC rSIF & $\begin{array}{c}\text { AUC GI } \\
\text { disso }\end{array}$ \\
\hline Compound A & 10 & 16.51 & 0.186 & 1.08 & 0.407 & 5.05 & \\
\hline Pred. vs. obs & & & 0.0113 & 0.0654 & 0.0247 & 0.3059 & \\
\hline Compound B & 3 & 1.928 & 0.146 & 0.146 & 0.414 & 1.867 & \\
\hline Pred. vs. obs & & & 0.0757 & 0.0757 & 0.2147 & 0.9684 & \\
\hline Compound C & 3 & 0.232 & 0.064 & 0.069 & 0.070 & 0.071 & \\
\hline Pred. vs. obs & & & 0.2759 & 0.2974 & 0.3017 & 0.3060 & \\
\hline Compound D & 3 & 2.631 & 0.570 & 0.607 & 1.154 & 1.570 & 1.802 \\
\hline Pred. vs. obs & & & 0.2166 & 0.2307 & 0.4386 & 0.5967 & 0.6849 \\
\hline Compound E & 10 & 26.4 & 0.758 & 0.795 & 4.803 & 9.351 & 9.335 \\
\hline Pred. vs. obs & & & 0.0287 & 0.0301 & 0.1819 & 0.3542 & 0.3536 \\
\hline Compound F & 10 & 13.4 & 7.812 & 7.812 & 7.823 & 7.812 & 7.734 \\
\hline Pred. vs. obs & & & 0.5830 & 0.5830 & 0.5838 & 0.5830 & 0.5772 \\
\hline Compound G & 3 & 0.131 & 0.020 & 0.116 & 0.090 & 0.285 & 0.260 \\
\hline Pred. vs. obs & & & 0.1527 & 0.8855 & 0.6870 & 2.176 & 1.985 \\
\hline Compound H & 3 & 1.06 & 0.007 & 0.007 & 0.228 & 0.312 & 0.546 \\
\hline Pred. vs. obs & & & 0.0066 & 0.0066 & 0.2151 & 0.2943 & 0.5151 \\
\hline Compound I & 3 & 0.571 & 0.038 & 0.033 & 0.062 & 0.19 & \\
\hline Pred. vs. obs & & & 0.0665 & 0.0578 & 0.1086 & 0.3327 & \\
\hline
\end{tabular}

When the in vitro equilibrium solubility in FaSSIF is used to correct the bile salt dependent solubility, an improved prediction is found in a number of cases. In order to allow for an estimate of bile salt dependent solubility in case FaSSIF solubility data is not available, the use of the theoretical bile solubilization ratio may be an option. As shown in Table 4 the results of this method might significantly deviate from the measured values. As the results in Table 6 and Table 7 suggest, for most of the investigated compounds the use of the theoretical bile salt ratio leads to an improved prediction, but underprediction for low solubility compounds can still be significant.

When comparing the simulation results that were generated using the in vitro rat SIF solubility, for nearly all compounds of the current test set an improved prediction result is achieved. This is reflected in a maximum deviation of the predicted value vs the observed value by a factor of at least 3 . For some compounds even the range of 2-fold variation is reached. Overprediction was observed only in one case (compound G), for which even FaSSIF-V2 solubility resulted in a too high value of $C_{\max }$.

In case of compound $\mathrm{D}$ and $\mathrm{H}$, the full $\mathrm{Gl}$ dissolution further improves the simulation outcome. However, taking into account the time and effort to record the dissolution profile, it appears justified to use rSIF equilibrium solubility instead. 
a)

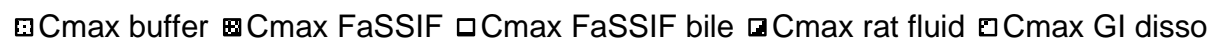

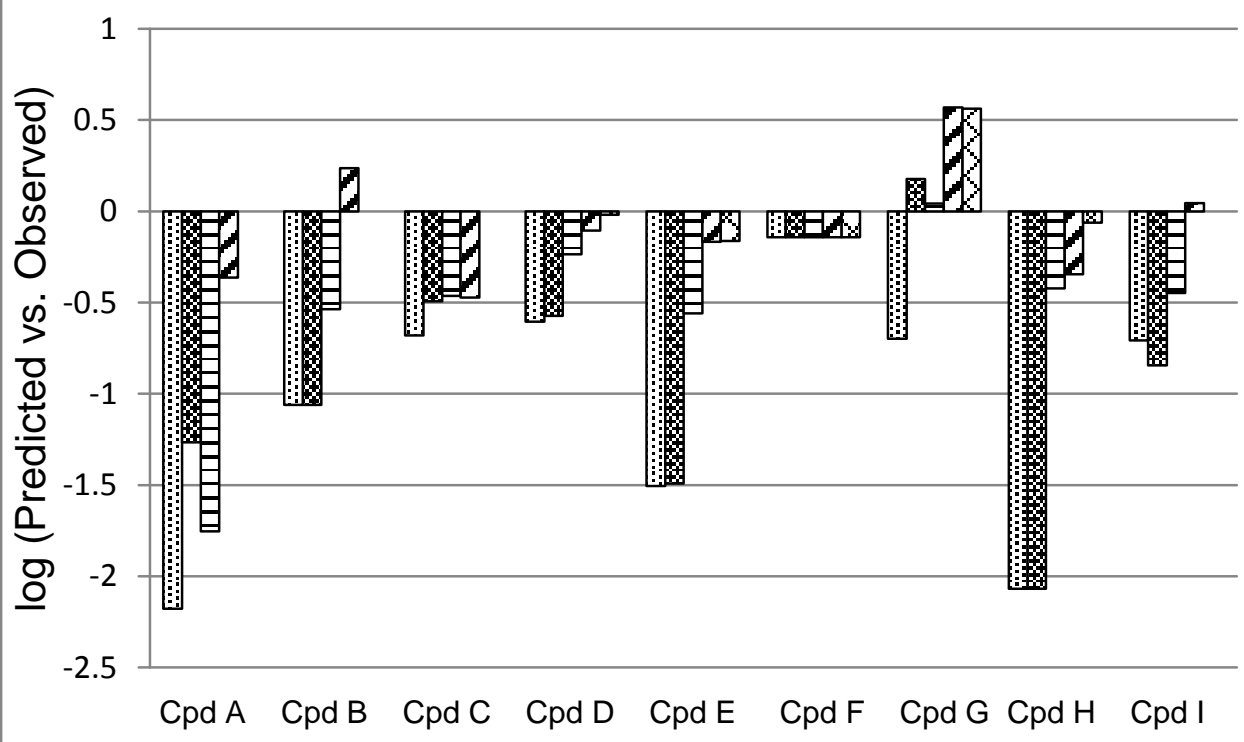

b)

口AUC buffer $\quad$ AUC FaSSIF $\boxminus A U C$ FaSSIF bile $\square$ AUC rat fluid $\square$ AUC GI disso

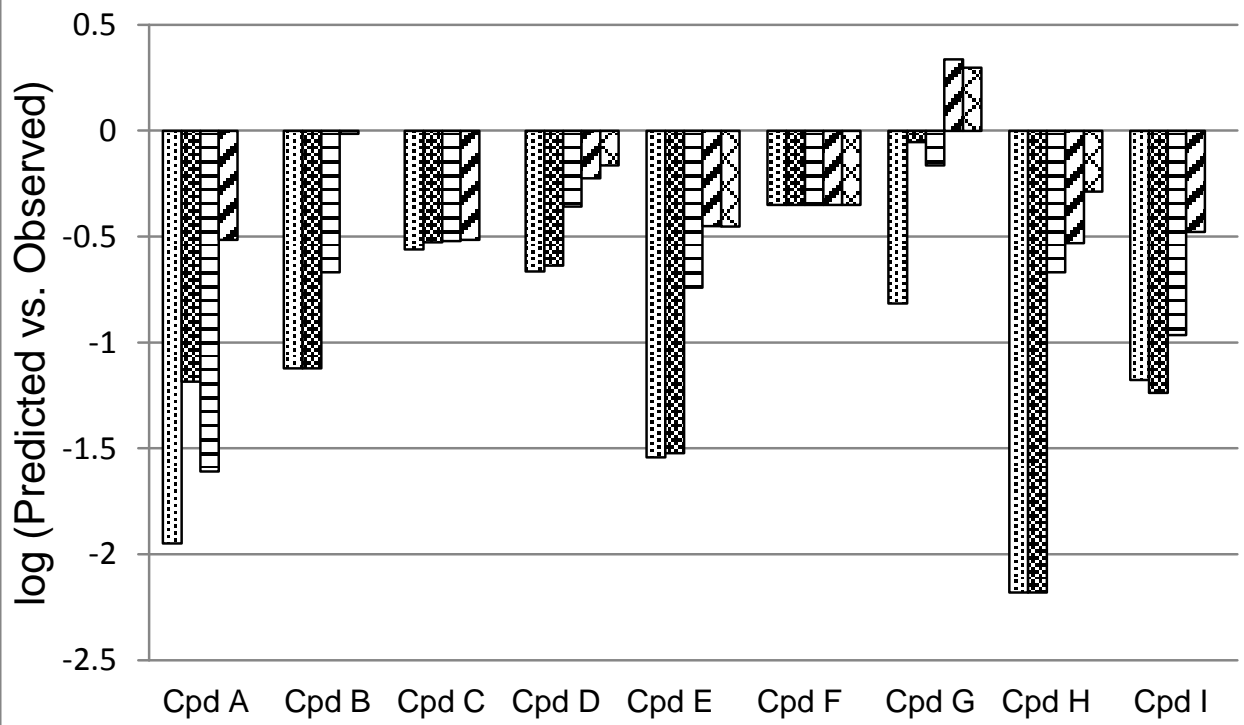

Figure 4. a) $C_{\max }$ and b) AUC from simulations performed on studies comprising doses from a 3 to $10 \mathrm{mg} / \mathrm{kg}$ dose

Observations at higher p.o. doses

Compared to low p.o. doses, the solubility and dissolution properties of a compound are assumed to impact the overall PK profile in an even stronger way when higher doses area applied.

PBPK results for doses from 30 to $300 \mathrm{mg} / \mathrm{kg}$ are summarized in Table 8 and Table 9. Figure 5 displays the results as ratio of predicted vs. observed for both $C_{\max }$ and AUC. Based on rSIF solubility, predicted $C_{\max }$ 
of all compounds tested is found within a 3-fold range as compared to observed values, most of them even within 2 -fold. The difference to all other equilibrium solubility values investigated is even more pronounced and full GI dissolution does not necessarily help improving the quality of PBPK prediction. The example of compound $\mathrm{F}$ reveals, again, that there is no influence of solubility for this compound, as already pointed out for the lower dose studies.

Table 8. PBPK results of $C_{\max }$ in $\mu \mathrm{g} / \mathrm{mL}$ for higher dose range based on various solubility input. $C_{\max }$ buffer, FaSSIF, FaSSIF with theoretical bile salt correction (Cmax FaSSIF bile) and rSIF refer to equilibrium values, whereas GI disso comprises the input of the full GI dissolution profile. Pred. vs. obs. denotes the ratio of the predicted values vs. the observed $C_{\max }$.

\begin{tabular}{|l|c|c|c|c|c|c|c|}
\hline & $\begin{array}{c}\text { Dose, } \\
\mathrm{mg} / \mathrm{kg}\end{array}$ & $C_{\max }$ obs & $\begin{array}{c}C_{\max } \\
\text { buffer }\end{array}$ & $\begin{array}{c}C_{\max } \\
\text { FaSSIF }\end{array}$ & $\begin{array}{c}C_{\max } \\
\text { FaSSIF } \\
\text { bile }\end{array}$ & $\begin{array}{c}C_{\max } \text { rat } \\
\text { fluid }\end{array}$ & $\begin{array}{c}C_{\max } \mathrm{Gl} \\
\text { disso }\end{array}$ \\
\hline Compound $\mathrm{A}$ & 30 & 8.581 & 0.047 & 0.401 & 0.121 & 4.139 & \\
\hline Pred. vs. obs & & & 0.0055 & 0.0467 & 0.0141 & 0.4823 & \\
\hline Compound A & 100 & 18.3 & 0.042 & 0.468 & 0.127 & 4.02 & \\
\hline Pred. vs. obs & & & 0.0023 & 0.0256 & 0.0069 & 0.2197 & \\
\hline Compound B & 30 & 0.784 & 0.022 & 0.023 & 0.119 & 1.531 & \\
\hline Pred. vs. obs & & & 0.0280 & 0.0293 & 0.1518 & 1.953 & \\
\hline Compound C & 30 & 0.546 & 0.041 & 0.101 & 0.236 & 0.447 & 0.491 \\
\hline Pred. vs. obs & & & 0.0751 & 0.1850 & 0.4322 & 0.8187 & 0.8993 \\
\hline Compound D & 30 & 0.908 & 0.095 & 0.058 & 0.271 & 0.380 & 2.04 \\
\hline Pred. vs. obs & & & 0.1046 & 0.0639 & 0.2985 & 0.4185 & 2.2467 \\
\hline Compound E & 100 & 3.592 & 0.094 & 0.220 & 1.227 & 3.869 & 4.74 \\
\hline Pred. vs. obs & & & 0.0262 & 0.0612 & 0.3416 & 1.077 & 1.320 \\
\hline Compound F & 100 & 17.0 & 26.3 & 27.3 & 25.9 & 27.4 & 27.4 \\
\hline Pred. vs. obs & & & 1.547 & 1.610 & 1.524 & 1.612 & 1.612 \\
\hline Compound G & 30 & 0.813 & 0.005 & 0.055 & 0.038 & 0.274 & 0.263 \\
\hline Pred. vs. obs & & & 0.0062 & 0.0677 & 0.0467 & 0.3370 & 0.3235 \\
\hline
\end{tabular}

Prediction of AUC in the dose range 30 to $300 \mathrm{mg} / \mathrm{kg}$ follows the same trend as for $C_{\max }$. rSIF solubility, besides the GI dissolution assay, provides best matches of PBPK results without a tendency to overpredict. For instance, for compound $B$ at a dose of $3 \mathrm{mg} / \mathrm{kg}$, without using the rSIF solubility but the calculated solubilization ratio an under-prediction of AUC by a factor of 5 was found. At the dose of $30 \mathrm{mg} / \mathrm{kg}$, the under-prediction was nearly 10 -fold, whereas the simulation based on rat SIF fluid led to an excellent match for AUC with a factor of 1.3-fold.

There are still results where AUC is underpredicted by a factor of more than five. One may hypothesize that for these cases, the impact of other parameters than solubility, like first pass extraction, might be more important in limiting exposure.

Our results obtained with nine compounds and across different dose ranges show that the rSIF solubility can be regarded as a very useful biorelevant solubility parameter when performing PBPK simulations for rats. 
Table 9. PBPK results of AUC (0-t) in $\mu \mathrm{g} \mathrm{h} / \mathrm{mL}$ for higher dose range based on various solubility input. AUC buffer, FaSSIF, FaSSIF with theoretical bile salt correction (AUC FaSSIF bile) and rSIF refer to equilibrium values, whereas GI disso comprises the input of the full GI dissolution profile. Pred. vs. obs. denotes the ratio of the predicted values vs. the observed AUC.

\begin{tabular}{|l|c|c|c|c|c|c|c|}
\hline & $\begin{array}{c}\text { Dose, } \\
\mathrm{mg} / \mathrm{kg}\end{array}$ & AUC obs & $\begin{array}{c}\text { AUC } \\
\text { buffer }\end{array}$ & $\begin{array}{c}\text { AUC } \\
\text { FaSSIF }\end{array}$ & $\begin{array}{c}\text { AUC } \\
\text { FaSSIF } \\
\text { bile }\end{array}$ & $\begin{array}{c}\text { AUC rat } \\
\text { fluid }\end{array}$ & $\begin{array}{c}\text { AUC GI } \\
\text { disso }\end{array}$ \\
\hline Compound A & 30 & 41.3 & 0.514 & 2.057 & 0.861 & 15.2 & \\
\hline Pred. vs. obs & & & 0.0124 & 0.0498 & 0.0209 & 0.3680 & \\
\hline Compound A & 100 & 97.5 & 0.297 & 2.48 & 0.766 & 19.2 & \\
\hline Pred. vs. obs & & & 0.0030 & 0.0254 & 0.0079 & 0.1969 & \\
\hline Compound B & 30 & 11.4 & 0.274 & 0.274 & 1.274 & 14.75 & \\
\hline Pred. vs. obs & & & 0.0240 & 0.0240 & 0.1118 & 1.2939 & \\
\hline Compound C & 30 & 6.99 & 0.175 & 0.317 & 0.521 & 0.706 & 0.874 \\
\hline Pred. vs. obs & & & 0.0250 & 0.0454 & 0.0745 & 0.1010 & 0.1250 \\
\hline Compound D & 30 & 14.83 & 1.139 & 0.707 & 2.900 & 4.27 & 18.02 \\
\hline Pred. vs. obs & & & 0.0768 & 0.0477 & 0.1955 & 0.2879 & 1.215 \\
\hline Compound E & 100 & 56.9 & 1.14 & 4.80 & 15.1 & 35.8 & 44.0 \\
\hline Pred. vs. obs & & & 0.0200 & 0.0844 & 0.2654 & 0.6292 & 0.7733 \\
\hline Compound F & 100 & 268 & 78.0 & 78.1 & 78.2 & 78.1 & 78.1 \\
\hline Pred. vs. obs & & & 0.2910 & 0.2914 & 0.2918 & 0.2914 & 0.2914 \\
\hline Compound G & 30 & 6.25 & 0.033 & 0.279 & 0.202 & 1.249 & 1.225 \\
\hline Pred. vs. obs & & & 0.0052 & 0.0446 & 0.0323 & 0.1998 & 0.1960 \\
\hline
\end{tabular}




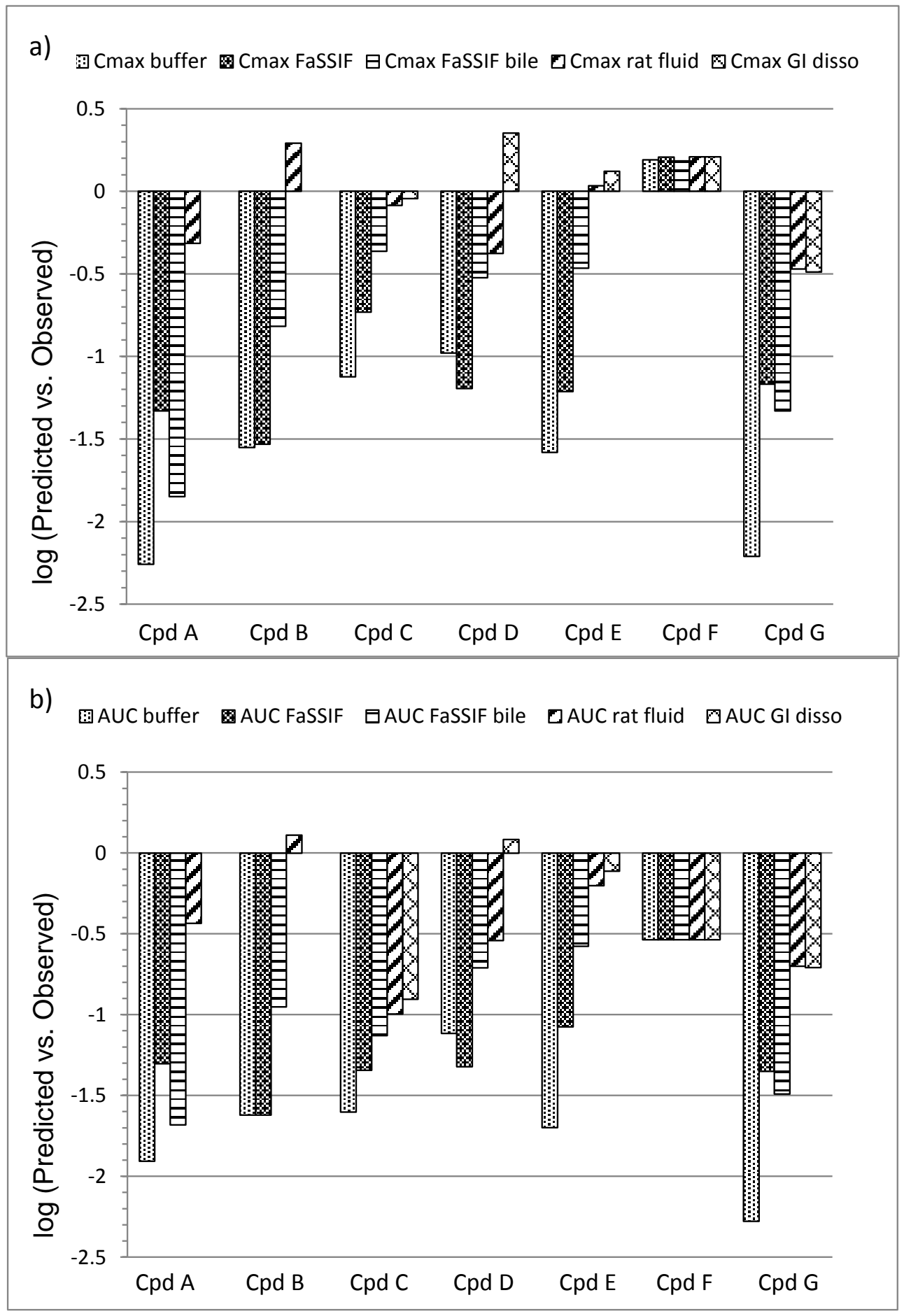

Figure 5. Observed a) $C_{\max }$ and b) $A U C$ results from simulations performed on studies comprising doses from a 30 to $300 \mathrm{mg} / \mathrm{kg}$ dose

\section{Conclusions}

Biorelevant solubility parameters can be markedly different across species. For proper prediction of solubility mediated exposure limitation in human, one needs to understand the solubility limitation across in vivo species. A good exposure in rats will not necessarily translate into acceptable human drug absorption, if solubility is mainly driven by bile salt solubilization, or may lead to an undesired absorption profile, like a strong food effect. Even good exposure in a non-rodent species, like dog, might not be indicative of the situation in humans, as the dog intestinal fluid still contains bile salts at a level that is close to the post-prandial situation in humans. 
A better understanding of the impact of solubility when drug absorption is studied in various species helps to lower the risk of failure in clinical studies. For this, a rat simulated intestinal fluid (rSIF) is proposed to support understanding the role that solubility plays for absorption of a particular drug candidate.

The proposed rat SIF greatly improves the quality of PBPK predictions in rats. It explains why some compounds that are low soluble in aqueous media nevertheless show good p.o. exposure in rats and why this might not always translate to higher species. The results presented in this study allow for a better prediction of exposure in rats across the dose range of interest. In addition, a better understanding of solubility mediated exposure in rats will help to better anticipate the risk of solubility limited exposure in higher species and ultimately in humans.

\section{Acknowledgements}

The authors gratefully thank Daniel Gosling and Philippe Monbaron for performing the solubility and dissolution experiments. Further we greatly acknowledge the contribution of Angus Hibbins to the development of rSIF.

\section{References}

[1] E.S. Kostewicz, L. Aarons, M. Bergstrand, M.B. Bolger, A. Galetin, O. Hatley, M. Jamei, R. Lloyd, X. Pepin, A. Rostami-Hodjegan, E. Sjögren, C. Tannergren, D.B. Turner, C. Wagner, W. Weitschies, J. Dressman, European Journal of Pharmaceutical Sciences 57 (2014) 300-321.

[2] E.L. McConnel, A.W Basit, S. Murdan, The Journal of Pharmacy and Pharmacology 60(1) (2008) 6370.

[3] R. Holm, A. Mullertz, H. Mu, International Journal of Pharmaceutics 453(1) (2013) 44-55.

[4] G. Amidon, H. Lennernäs, V. Shah, J. Crison, Pharmaceutical Research 12(3) (1995) 413-420.

[5] H. Jones, N. Parrott, G. Ohlenbusch, T. Lavé, Clinical Pharmacokinetics 45(12) (2006) 1213-1226.

[6] Y. Gao, R.A. Carr, J.K. Spence, W.W. Wang, T.M. Turner, J.M. Lipari, J.M. Miller, Molecular Pharmaceutics 7(5) (2010) 1516-1526.

[7] M. Hagio, M. Matsumoto, M. Fukushima, H. Hara, S. Ishizuka, Journal of Lipid Research 50(1) (2009) 173-180.

[8] J. M. Dietschy, Journal of Lipid Research 9(3) (1968) 297-309.

[9] Y. Tanaka, T. Hara, W. Ryoichi, N. Shunji, Journal of Pharmacy \& Pharmaceutical Sciences 15(4) (2012), 510-518.

[10] H. Musther, A. Olivares-Morales, O.J. Hatley, B. Liu, A. Rostami Hodjegan, European Journal of Pharmaceutical Sciences 57 (2014) 280-291.

[11] M. Arndt, H. Chokshi, K. Tang, N.J. Parrott, C. Reppas, J.B. Dressman, European Journal of Pharmaceutics and Biopharmaceutics 84(3) (2013) 633-641.

[12] N. Parrott, N. Paquereau, P. Coassolo, T. Lave, Journal of Pharmaceutical Sciences 94(10) (2005) 2327-2343.

[13] F. Wohnsland, B. Faller, Journal of Medicinal Chemistry 44(6) (2001) 923-930.

[14] E. Jantratid, N. Janssen, C. Reppas, J.B. Dressman, Pharmaceutical Research 25(7) (2008) 1663-1676.

[15] K. Magwedere, S. Mukaratirwa, International Journal of Applied Research in Veterinary Medicine 6(3) (2008) 166-174.

[16] J.M. DeSesso, A.L. Williams, Annual Reports in Medicinal Chemistry 43 (2008) 353-371.

[17] T.T. Kararli, Biopharmaceutics \& Drug Disposition 16 (1995) 351-380.

[18] D.M. Heuman, Z.R. Vlahcevic, M.L. Bailey, P.B. Hylemon, Hepatology 8(4) (1988) 892-897. 
[19] H.A. Merchant, S.C. Rabbie, F.J.O. Varum, F. Afonso-Pereira, A.W. Basit, European Journal of Pharmaceutical Sciences 62 (2014) 76-85.

[20] S.D Mithani, V. Bakatselou, C.N. TenHoor, J. B. Dressman, Pharmaceutical Research 13(1) (1996) 163167.

[21] M. Germani, P. Crivori, M. Rocchetti, P.S. Burton, A.G. Wilson, M.E. Smith, I. Pogessi, European Journal of Pharmaceutical Sciences 31 (2007) 190-201.

[22] H. M. Jones, I. B. Gardner, K. J. Watson, The AAPS Journal 11(1) (2009) 155-166.

(C)2016 by the authors; licensee IAPC, Zagreb, Croatia. This article is an open-access article distributed under the terms and conditions of the Creative Commons Attribution license (http://creativecommons.org/licenses/by/3.0/) (cc)) EY 